\title{
Severe Outcome of Pharyngeal-Cervical-Brachial Pure Motor Axonal Neuropathy
}

\author{
L. Bonanni*, ${ }^{,}$V. Onofrj ${ }^{2}$, V. Scorrano ${ }^{3}$, M. Onofrj ${ }^{1}$ and A. Thomas ${ }^{1}$ \\ ${ }^{I}$ Department of Neurology, University G.D'Annunzio of Chieti-Pescara and Aging Research Center, Ce.S.I., "Gabriele \\ d'Annunzio" University Foundation, Italy \\ ${ }^{2}$ University Ospedale San Raffaele, Milan, Italy \\ ${ }^{3}$ Neurofisiopatologia, Ospedale Civile di Pescara, Italy
}

\begin{abstract}
We present two further cases of the pharyngeal-cervical-brachial (PCB) form of GBS, with unfavourable outcome, showing dramatic dissociation between upper and lower body Symptoms.

Both patients showed rapidly progressive motor denervation with disappearance of Compound Muscle Action Potentials (CMAPs) in upper limbs muscles. Sensory Nerve Action Potentials (SNAPs) were instead normal. Normal reflexes, F waves and action potentials were elicited in lower limbs. Despite i.v. Immunoglobulin treatment no recovery was observed and both patients died within a year from onset of symptoms.
\end{abstract}

\section{INTRODUCTION}

Guillain-Barré Syndrome(GBS) is probably an autoimmune disorder with peripheral nerve involvement [1] characterized in its usual presentation by widespread muscle weakness with areflexia, ascending from lower limbs(Landry's Ascending Paralysis) [2].

However, GBS includes variants presenting with focal signs and symptoms $[3,4]$ and prominently axonal or demyelinating forms [5].

Among them, a pharyngeal-cervical-brachial (PCB) form was described by Ropper in 1986 [6] who described three cases of pharyngeal-cervical-brachial weakness. Preservation of power and reflexes and normal electrophysiological parameters in the lower limbs are common features of PCB eventhough mild weakness of lower limbs may be present [6].

We present two cases of PCB with severely unfavourable evolution, with pure axonal motor degeneration.

\section{CASE REPORT}

1. A 72y.o. man was admitted to the hospital for the appearance, 15 days after an upper respiratory tract infection, of rhinolalia, difficulty in swallowing, upper limbs weakness, rapidly progressing to dysarthria, severe dysphagia and respiratory deficit, making necessary tube feeding and assisted ventilation. Body temperature was normal. Patient was wakeful and aware. Chest x-ray showed bilateral diaphragmatic paresis. Brain and cervical-spine MRI scan was normal.

\footnotetext{
*Address correspondence to this author at the Department of Neurology, University G.D'Annunzio of Chieti-Pescara and Aging Research Center, Ce.S.I., "Gabriele d'Annunzio" University Foundation, Italy;

E-mail: 1.bonanni@unich.it
}

Eye movements and pupillary light reflex were normal. Sphincteric control was preserved but the patient was bladder catheterised as a routinal practice in critical care unit. Patient presented deficit of the eye-lid and lip closure. Muscle strength (Medical Research Council scale) was 2 in the neck flexors, 1-2 in distal and 2-3 in proximal muscles of the upper limbs; strength was slightly impaired (4) in the left quadriceps [7] and normal in the right leg and feet. Patient was areflexic in upper limbs, tendon reflexes were normal in lower limbs. Pathological reflexes, sensory/vegetative disturbances, lower limb ataxia were absent.

At day 7 from symptoms onset, muscle strength was 1 in the neck flexors, 0 in distal and 1 in proximal muscles of the upper limbs. No variation of strength was evidenced in lower limbs. At day 15 muscle strength was 0 in the neck flexor and the upper limbs were completely plegic ( 0 in proximal and distal muscles).

Laboratory tests including glucose, liver and muscle enzymes and electrolytes, seral protein levels were normal. Serum sampled on the day of admission showed no evidence of botulinum toxin (neutralization bioassay) or of anti-CMV . Anti-Campylobacter jejuni IgM and IgG (enzyme-linked immunosorbent assay, ELISA) were positive. Diphteria was excluded because patient had received vaccination and bacterial cultures were negative. CSF examination at onset showed normal findings with no cells and normal protein level $(25 \mathrm{mg} / \mathrm{dl}$, upper normal limit $40 \mathrm{mg} / \mathrm{dl})$; at day 15 modest increase in protein level $(55 \mathrm{mg} / \mathrm{dl})$ and no cells were detected.

We measured serum IgG antibodies to GT1a, GQ1b, GM1, GM1b, GM2, GD1a, GalNAc-GD1a, GD1b, and GT1b by ELISA. None of the antibodies tested was resulted positive.

At onset electrophysiology showed modest reduction of CMAP amplitude (1.5-2 uV from hand muscles), normal 
conduction velocity, modest increment of the distal motor latency registered from hand muscles $(4.5 \mathrm{~ms}$ median nerve, $3.7 \mathrm{~ms}$ ulnar nerve, normal limits 5.0-2.5). F waves could not be regularly elicited from hand muscles; rare motor unit potentials were recruited during voluntary activity; findings were normal in lower limbs; Sensory Nerve Action Potentials (SNAPs) were normal in latency and amplitude in both upper and lower limbs.

At day 15 CMAPs could not be elicited in upper limbs or diaphragm. Spontaneous activity with fibrillation potentials and positive sharp waves (PSW) was diffusely present in upper limbs. Somatosensory evoked potentials(SSEPs) were normal.

Patient was treated with i.v. Immunoglobulin $(2 \mathrm{~g} / \mathrm{Kg})$ for 2days, according to a widely accepted protocol [8] at day 2 and at day 15 from symptom onset.

After one month from clinical onset, dysphagia, dysartrhia and upper limb weakness showed no recovery. Tendon reflexes were absent in upper limbs. Electrophysiology showed absent motor response to electrical stimulation with massive denervation in the upper limbs and diaphragm, no voluntary activity. No change in lower limbs and sensory components.

Six month after the onset the patient was stable with no further improvement of muscle weakness. He was still on assisted ventilation and tube feeding and was referred to a sub-intensive care unit. In the following year the patient died because of cardiopulmonary insufficiency following generalized sepsis.

2. A 68 y.o. man was admitted to our hospital for the appearance 10 days after a gastrointestinal infection of weakness in both hands and dysarthria. MRI scan at admission was normal. After one day acute respiratory failure and dysphagia occurred requiring tracheotomy and tube feeding. Chest X-ray showed bilateral diaphragmatic paresis.

Eye and facial muscles movements were normal. Strength was 0 in right deltoid, 2 in the right biceps and triceps, 0 in the muscles of the left upper limb; normal in lower limbs. Tendon reflexes were absent in upper limbs, normal in lower limbs. No pathological reflexes, sensory/vegetative signs nor lower limb ataxia were present. Neurological examination after 2 weeks from treatment showed no improvement of symptoms.

CSF examination at admission showed normal protein level of $28 \mathrm{mg} / \mathrm{dl}$ and 3 cells; at day 15 from clinical onset CSF showed 3 cells and protein levels of $64 \mathrm{mg} / \mathrm{dl}$.

Laboratory tests including glucose, liver and muscle enzymes and electrolytes, seral protein levels were normal. Diphteria was excluded because patient was vaccinated and bacterial cultures were negative. Botulinum toxin assay was negative. CMV serology was negative; Campylobacter jejuni IgM were positive. Low anti-GT1a IgG activity was found with negativity for anti-GD1a IgG and anti-GQ1b IgG.

Motor Nerve test at admission showed modest reduction of CMAP amplitude (0.9-1.7 ( V from hand muscles), slight increment of the distal motor latency in left median nerve $(4.2 \mathrm{~ms})$; normal conduction velocity and normal latency and amplitude of SNAPs in upper limbs. Voluntary activity elicited sporadic MUAPs. Normal findings in lower limbs.
At day 15 electrophysiology showed absence of CMAP in upper limbs with inexcitability of all upper limb nerves and normal SNAPs, absence of voluntary activation, diffuse PSWs and fibrillation potentials in upper limb and diaphragm. Electrophysiological findings were normal in lower limbs.

SSEPs were normal. Patient was treated with i.v. Immunoglobulins at the dose of $2 \mathrm{~g} / \mathrm{Kg} /$ day for 2 days at day 3 from symptoms onset and at day 15 with no improvement of symptoms.

After one month, neurological examination showed no recovery of bulbar palsy. Slight improvement of weakness in the right upper limb ( 1 in deltoid, 2 in biceps and triceps, 0 in distal hand muscles). No improvement of left upper limb muscle strength, with inexcitability of all nerves tested and diffuse denervation of upper limb muscles.

At six months from the onset clinical and electrophysiological signs were stable. Patient was on assisted ventilation in a sub-intensive care unit, he died after one year after an episode of pneumonia.

Patient caregivers signed an informed consent allowing us to videotape the patients.

Tables $\mathbf{1}$ and $\mathbf{2}$ summarize electrophysiological recordings in comparison with normative data for both patients.

\section{DISCUSSION}

We presented two cases of pure motor PCB with very unfavourable outcome.

Bulbar palsy was the initial symptom as described by Ropper; involvement of upper limbs was subsequent. Lower limb strength and reflexes were spared with normal EMG parameters, SNAPs were normal, CMAPs in upper limbs completely disappeared in two weeks. Patients were on assisted ventilation 6 months after the onset, despite i.v. Ig treatment. In both cases absent motor recovery led to fatal outcome.

In both patients, therefore, electrophysiological recording at onset and in the following weeks showed pure axonal motor neuropathy progressing to complete denervation of upper limb muscles and diaphragm, with electrical inexcitability of muscles, while SNAPs in upper limbs were normal throughout follow up.

In previous literature partial recovery was described in 11 cases $[6,9,10]$, even though all reports evidenced prominent or pure axonal motor forms. Serum antibody studies showed, same as in our patients, absence of anti-GQ1b, considered typical of Miller Fisher Syndrome (MFS) [11]. Some reports suggest that GT1a could be associated with the PCB form of axonal GBS $[7,10,12]$. Only in one of our patients (case 2), we found positivity for Anti-GT1a antibodies.

At difference with previous reports of PCB [10] in both our cases the dissociation between upper and lower limb involvement was striking with complete strength loss in upper limbs after 15 days from onset. The complete sparing of strength and CMAPs in lower limbs, and complete sparing of SNAPs in upper limbs was also dramatic.

Our patients did not respond to i.v. IgG treatment which has been described to be at least partially beneficial in PCB [10]. 
Table 1. Electrophysiological Characteristics of Case 1 at Symptom Onset and After 15 Days

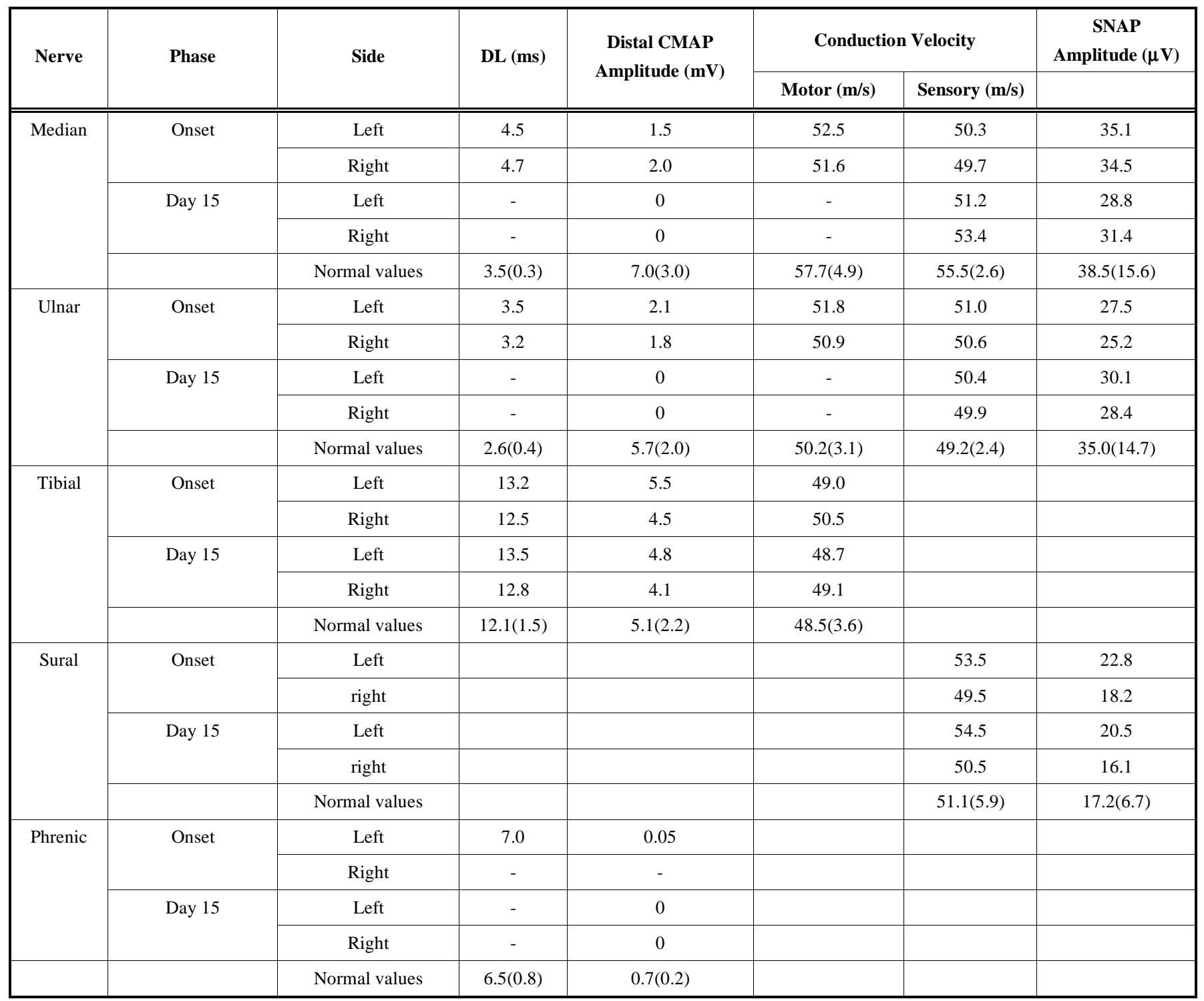

Normal values are given as mean(SD).

Table 2. Electrophysiological Characteristics of Case 2 at Symptom Onset and After 15 Days

\begin{tabular}{|c|c|c|c|c|c|c|c|}
\hline \multirow[t]{2}{*}{ Nerve } & \multirow[t]{2}{*}{ Phase } & \multirow[t]{2}{*}{ Side } & \multirow[t]{2}{*}{ DL (ms) } & \multirow{2}{*}{$\begin{array}{c}\text { Distal CMAP } \\
\text { Amplitude (mV) }\end{array}$} & \multicolumn{2}{|c|}{ Conduction Velocity } & \multirow{2}{*}{$\begin{array}{c}\text { SNAP } \\
\text { Amplitude }(\mu \mathrm{V})\end{array}$} \\
\hline & & & & & Motor (m/s) & Sensory $(\mathbf{m} / \mathbf{s})$ & \\
\hline \multirow{3}{*}{ Median } & Onset & Right & 4.5 & 2.0 & 58.6 & 51.7 & 30.5 \\
\hline & \multirow[t]{2}{*}{ Day 15} & Left & - & 0 & & 55.2 & 27.8 \\
\hline & & Normal values & $3.5(0.3)$ & $7.0(3.0)$ & $57.7(4.9)$ & $55.5(2.6)$ & $38.5(15.6)$ \\
\hline \multirow[t]{3}{*}{ Ulnar } & \multirow[t]{2}{*}{ Onset } & Left & 3.5 & 1.8 & 55.0 & 50.8 & 215 \\
\hline & & Right & 3.8 & 2.0 & 57.7 & 51.6 & 22.6 \\
\hline & Day 15 & Left & - & 0 & 0 & 50.4 & 30.1 \\
\hline
\end{tabular}


(Table 2). Contd.....

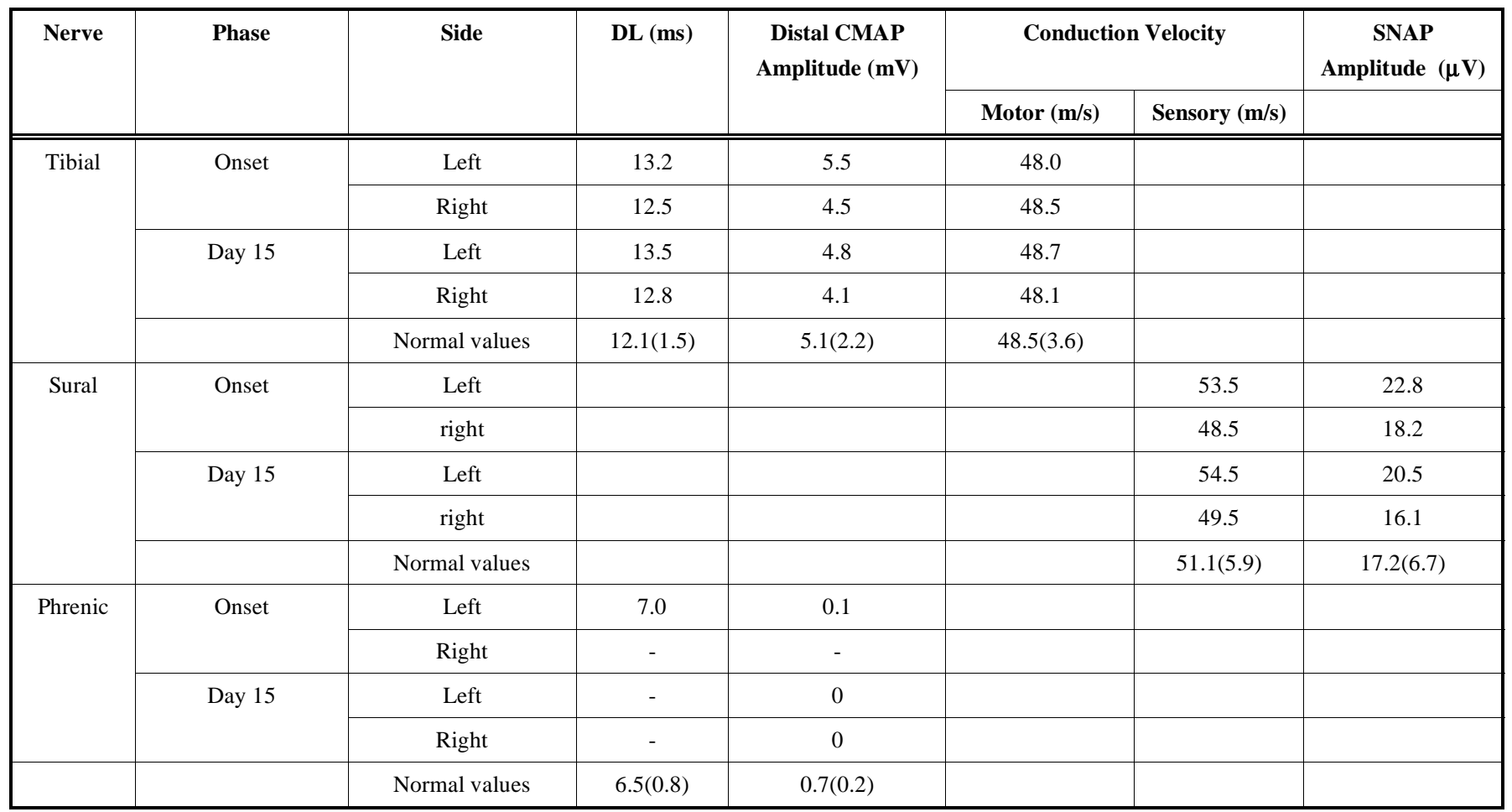

Normal values are given as mean(SD).

\section{ACKNOWLEDGEMENTS}

Publication expenses were covered by University G.d'Annunzio of Chieti-Pescara, Italy.

\section{REFERENCES}

[1] Asbury AK, Cornblath DR. Assessment of current diagnostic criteria for Guillain-Barrés Syndrome. Ann Neurol 1990; 27: S214.

[2] Landry JBO. Note sur la paralysie ascendante aigue. Gazette hebdomadaire de médecine et de chirurgie 1859; 6: 472-4,486-8.

[3] Guillain G. Les polyradiculonevrites avec dissociation albuminocytologique et à evolution favorable. J Belge Neurol Psychiatry 1938; 38: 323-9.

[4] Fisher M. An unusual variant of acute idiopathic polyneuritis (syndrome of ophthalmoplegia, ataxia, and areflexia). N Engl J Med 1956; 255: 57-65.

[5] Levin KH. Variants and mimics of Guillain Barré syndrome. The Neurologist 2004; 10: 61-74.

[6] Ropper AH. Unusual clinical variants and signs in Guillain-Barré Syndrome. Arch Neurol 1986; 43: 1150-2.
[7] Mizoguchi K, Hase A, Obi T, et al. Two species of antiganglioside antibodies in a patient with pharyngeal-cervical-brachial variant of Guillain Barré syndrome. J Neurol Neurosurg Psychiatry 1994; 57: 1121-3.

[8] Hughes RAC, Wijdicks EFM, Barohn R, et al. Practice parameter: immunotherapy for Guillain-Barré syndrome. Report of the Quality Standards Subcommittee of the American Academy of Neurology. Neurology 2003; 61: 736-40.

[9] Arai M, Susuki K, Koga M. Axonal pharyngeal-cervical-brachial variant of Guillain-Barre'syndrome without anti-GT1a IgG antibody. Muscle Nerve 2003; 28: 246-50.

[10] Murakami N, Tomita Y, Koga M, et al. An adolescent with pharyngeal-cervicalbrachial variant of Guillain Barré sindrome after cytomegalovirus infection. Brain Dev 2006; 28: 269-71.

[11] Yuki N, Sato S, Tsuji S, et al. Frequent presence of anti-GQ1b antibody in Fisher's syndrome. Neurology 1993; 43: 414-7.

[12] Nagashima T, Koga M, Odaka M, Hirata K, Yuki. Clinical correlates of serum anti-GT1a IgG antibodies. J Neurol Sci 2004; 219: $139-45$.

(C) Bonanni et al.; Licensee Bentham Open.

This is an open access article licensed under the terms of the Creative Commons Attribution Non-Commercial License (http://creativecommons.org/licenses/by-nc/3.0/) which permits unrestricted, non-commercial use, distribution and reproduction in any medium, provided the work is properly cited. 